\title{
OSCILLATIONS OF THE SOLUTIONS OF NONLINEAR HYPERBOLIC EQUATIONS OF NEUTRAL TYPE
}

\author{
D.P. MisheV and D.D. Bainov
}

\begin{abstract}
In this paper nonlinear hyperbolic equations of neutral type of the form

$$
\begin{aligned}
\frac{\partial^{2}}{\partial t^{2}}[u(x, t) & +\lambda(t) u(x, t-\tau)]-[\Delta u(x, t)+\mu(t) \Delta u(x, t-\sigma)] \\
& +c(x, t, u)=f(x, t), \quad(x, t) \in \Omega \times(0, \infty) \equiv G,
\end{aligned}
$$

are considered, where $\tau, \sigma=$ const $>0$, with boundary conditions

or

$$
\frac{\partial u}{\partial n}+\gamma(x, t) u=g(x, t), \quad(x, t) \in \partial \Omega \times[0, \infty)
$$

$$
u=0, \quad(x, t) \in \partial \Omega \times[0, \infty) .
$$

Under certain constraints on the coefficients of the equation and the boundary conditions, sufficient conditions for oscillation of the solutions of the problems considered are obtained.
\end{abstract}

\section{Introduction}

In the last few years results related to the oscillatory properties and asymptotic behaviour of the solutions of some classes of hyperbolic cquations were published. We shall mention especially the work of $K$. Kreith, $T$. Kusano and N. Yoshida [4] in which sufficient conditions for oscillation of the solutions of the nonlinear hyperbolic equation

$$
u_{t i}-\Delta u+c(x, t, u)=f(x, t)
$$

considered in a cylindrical domain are obtained. Oscillatory properties of the solutions of hyperbolic differential equations with a deviating argument were investigated in the works of D. Georgiou, K. Kreith [2], D. Georgiou [3]. Hyperbolic differential equations with maxima were investigated in the work of D. Mishev [6] and some conditions for oseillation of the solutions of hyperbolic equations of neutral type werc obtained by D. Mishev and D. Bainov in [7], [8].

The present investigation is supported by the Ministry of Culture, Science and Education of People's Republic of Bulgaria under Grant 61. 


\section{Preliminary notes}

In the present paper sufficient conditions for oscillation of the solutions of nonlinear hyperbolic equations of neutral type of the form

$$
\begin{aligned}
\frac{\partial^{2}}{\partial t^{2}}[u(x, t)+\lambda(t) u(x, t-\tau)]-[\Delta u(x, t)+\mu(t) \Delta u(x, t-\sigma)]+ \\
+c(x, t, u)=f(x, t), \quad(x, t) \in \Omega \times(0, \infty) \equiv G
\end{aligned}
$$

are obtained, where $\tau, \sigma=$ const $>0, \Delta u(x, t)=\sum_{i=1}^{n} u_{x_{i} x_{i}}(x, t)$ and $\Omega$ is a bounded domain in $\mathbb{R}^{n}$ with a piccewise smooth boundary.

Consider bournlary conditions of the form

$$
\begin{array}{rr}
\frac{\partial u}{\partial n}+\gamma(x, t) u=g(x, t), & (x, t) \in \partial \Omega \times[0, \infty) \\
u=0, & (x, t) \in \partial \Omega \times[0, \infty)
\end{array}
$$

We shall say that conditions $(\mathrm{H})$ are satisfied if the following conditions hold:

H1. $\lambda(t) \in C^{2}([0, \infty) ;[0, \infty))$,

$$
\mu(t) \in C([0, \infty) ; \mathbb{P})
$$

H2. $c(x, t, u) \in C(G \times \mathbb{R} ; \mathbb{R})$,

H3. $c(x, t,-u)=-c(x, t, u), \quad(x, t, u) \in G \times(0, \infty)$,

H4. $c(x, t, u) \geq p(t) \cdot h(u), \quad(x, t, u) \in G \times(0, \infty)$,

where $p(t)$ is a continuous and positive function in the interval $(0, \infty)$ and $h(u)$ is a continuous, positive and convex function in the same interval $(0, \infty)$

$$
\begin{aligned}
& \text { H5. } f(x, t) \in C(G ; \mathbb{R}) \\
& \text { H6. } g(x, t) \in C(\partial \Omega \times[0, \infty) ; \mathbb{R}) \\
& \text { H7. } \gamma(x, t) \in C(\partial \Omega \times[0, \infty) ;[0, \infty)) .
\end{aligned}
$$

Definition 1. The solution $u(x, t) \in C^{2}(G) \cap C^{1}(\bar{G})$ of problem (1), (2) (( $1,(3))$ is said to oscillate in the domain $G$ if for any positive number $\mu$ there exists a point $\left(x_{0}, t_{0}\right) \in \Omega \times[\mu, \infty)$, such that the cquality $u\left(x_{0}, t_{0}\right)=0$ holds.

In the subsequent theorems sufficient conditions for oscillation of the solutions of problems (1), (2) and (1), (3) in the domain $G$ are obtained. We shall note that in the work of K. Kreith, T. Kusano, N. Yoshida [4] conditions are obtained for the oscillation of the solutions only of problem (1), (2) in the casc when $\lambda(t) \equiv 0, \mu(t) \equiv 0$ and $\gamma(x, t) \equiv 0$. 
Introduce the following notation:

$$
\begin{array}{ll}
F(t)=\frac{1}{|\Omega|} \cdot \int_{\Omega} f(x, t) d x, & t>0 \\
G(t)=\frac{1}{|\Omega|} \cdot \int_{\partial \Omega} g(x, t) d s, & t>0
\end{array}
$$

where $|\Omega|=\int_{\Omega} d x$.

With any solution $u(x, t) \in C^{2}(G) \cap C^{1}(\bar{G})$ of problem (1), (2) we associate the function

$$
v(t)=\frac{1}{|\Omega|} \cdot \int_{\Omega} u(x, t) d x, \quad t>0 .
$$

Lemma 1. Let conditions (H) hold and let $u(x, t)$ be a positive solution of problem (1), (2) in the domain $G$. Then the function $v(t)$ defined by (5) satisfies the differential inequality of neutral type

(6) $\frac{d^{2}}{d t^{2}}[v(t)+\lambda(t) v(t-\tau)]+p(t) h(v(t)) \leqq G(t)+\mu(t) G(t-\sigma)+F(t)$,

$t \geqq t_{0}$, where $t_{0}$ is o suffciently large positive number.

Proof: Let $u(x, t)$ be a positive solution in the domain $G$ of problem (1), (2) and $t_{0}=\max \{\tau, \sigma\}$. Then $u(x, t-\tau)>0$ and $u(x, t-\sigma)>0$ for $(x, t) \in \Omega \times\left[t_{0}, \infty\right)$. We integrate both sides of equation (1) with respect to $x$ over the domain $\Omega$ and obtain for $t \geqq t_{0}$ :

$$
\begin{aligned}
& \frac{d^{2}}{d t^{2}}\left[\int_{\Omega} u(x, t) d x+\lambda(t) \int_{\Omega} u(x, t-\tau) d x\right]-\left[\int_{\Omega} \Delta u(x, t) d x+\right. \\
& \left.\quad+\mu(t) \int_{\Omega} \Delta u(x, t-\sigma) d x\right]+\int_{\Omega} c(x, t, u) d x=\int_{\Omega} f(x, t) d x
\end{aligned}
$$

From Green's formula and condition $\mathrm{H} 7$ it follows that

$$
\begin{aligned}
& \int_{\Omega} \Delta u(x, t) d x=\int_{\partial \Omega} \frac{\partial u}{\partial n} d s=\int_{\partial \Omega}[g(x, t)-\gamma(x, t) u] d s \leq \int_{\partial \Omega} g(x, t) d s \\
& \text { (9) } \quad \int_{\Omega} \Delta u(x, t-\sigma) d x=\int_{\partial \Omega} \frac{\partial u}{\partial n}(x, t-\sigma) d s= \\
& \quad=\int_{\partial \Omega}[g(x, t-\sigma)-\gamma(x, t-\sigma) \cdot u(x, t-\sigma)] d s \leqq \int_{\partial \Omega} g(x, t-\sigma) d s
\end{aligned}
$$


Moreover, from condition $\mathrm{H} 4$ and Jensen's inequality it follows that

$$
\begin{aligned}
& \int_{\Omega} c(x, t, u) d x \geqq p(t) \int_{\Omega} h(u(x, t)) d x \geqq \\
\geqq & p(t) h\left(\int_{\Omega} u(x, t) d x \cdot\left(\int_{\Omega} d x\right)^{-1}\right) \cdot \int_{\Omega} d x=p(t) \cdot h(v(t)) \cdot|\Omega|
\end{aligned}
$$

Using (8)-(10) and condition $\mathrm{H} 1$, from (7) we obtain

$$
\frac{d^{2}}{d t^{2}}[v(t)+\lambda(t) v(t-\tau)] \leqq G(t)+\mu(t) G(t-\sigma)+F(t)-p(t) \cdot h(v(t)),
$$

which proves Lemma 1.

\section{Main results}

Theorem 1. Let conditions $(H)$ hold and let the differential inequalities of neutral type

$$
\frac{d^{2}}{d t^{2}}[v(t)+\lambda(t) v(t-\tau)]+p(t) \cdot h(v(t)) \leqq G(t)+\mu(t) G(t-\sigma)+F(t)
$$

$$
\frac{d^{2}}{d t^{2}}[v(t)+\lambda(t) v(t-\tau)]+p(t) \cdot h(v(t)) \leqq G(t)-\mu(t) \cdot G(t-\sigma)-F(t)
$$

have no eventually positive solutions. Then each solution $u(x, t)$ of problem (1), (2) oscillates in the domain $G$.

Proof: Let $\mu>0$ be a positive number. Suppose that the assertion of the theorem is not true and let $u(x, t)$ be a solution of problem (1), (2) without zeroes in the domain $G_{\mu}=\Omega \times(\mu, \infty)$. If $u(x, t)>0$ for $(x, t) \in G_{\mu}$, then from Lemma 1 it follows that the function $v(t)$ defined by (5) is a positive solution of inequality (1I) for $t \geq t_{0}+\mu$, i.e it is an eventually positive solution of (11) which contradicts the assumption of the thoorem. If $u(x, t)<0$ for $(x, t) \in G_{\mu}$, then the function $-u(x, t)$ is a positive solution of the problem

$$
\left\{\begin{aligned}
\frac{\partial^{2}}{\partial t^{2}}[u+\lambda(t) u(x, t-\tau)]-[\Delta u & +\mu(t) \Delta u(x, t-\sigma)]+ \\
& +c(x, t, u)=-f(x, t),(x, t) \in G \\
\frac{\partial u}{\partial n}+\gamma(x, t) u=-g(x, t), \quad & (x, t) \in \partial \Omega \times[0, \infty) .
\end{aligned}\right.
$$


From Lemma 1 it follows that the function $\frac{1}{|\Omega|} \int_{\Omega}(-u(x, t)) d x$ is a positive solution of inequality (12) for $t \geqq t_{0}+\mu$ which also contradicts the assumption of the theorem. Thus Theorem 1 is proved.

Now we shall investigatge the oscillatory properties of the solutions of problem (1), (3). Consider in the domain $\Omega$ the following Dirichlet problem:

$$
\left\{\begin{array}{l}
\Delta U+\alpha U=0 \text { in } \Omega \\
\left.U\right|_{\partial \Omega}=0
\end{array}\right.
$$

where $\alpha=$ const. It is well known [1] that the smallest eigenvalue $\alpha_{0}$ is positive and the corresponding eigenfunction $\varphi(x)$ can be chosen to satisfy the inequality $\varphi(x)>0$ for $x \in \Omega$.

With any solution $u(x, t) \in C^{2}(G) \cap C^{1}(\bar{G})$ of problern (1), (3) we associate the function

$$
u(t)=\int_{\Omega} u(x, t) \varphi(x) d x \cdot\left(\int_{\Omega} \varphi(x) d x\right)^{-1}, \quad t>0
$$

We shall note that a similar averaging was frst used by $N$. Yoshida in the work [10].

Lemma 2. Let conditions H1-H6 hold and let $u(x, t)$ be a positive solution in the domain $G$ of problem (1), (3). Then the function $w(t)$ defined by (13) satisfies the differential inequality of neutral type

$$
\begin{aligned}
& \frac{d^{2}}{d t^{2}}[w(t)+\lambda(t) w(t-\tau)]+\alpha_{0} w(t)+\alpha_{0} \mu(t) \cdot w(t-\sigma)+ \\
+ & p(t) \cdot h(w(t)) \leqq \int_{\Omega} f(x, t) \varphi(x) d x \cdot\left(\int_{\Omega} \varphi(x) d x\right)^{-1}, \quad t \geqq t_{0},
\end{aligned}
$$

where $t_{0}$ is a sufficiently large positive number.

Proof: Let $u(x, t)$ be a positive solution in the domain $G$ of problem (1), (3) and $t_{0}=\max \{\tau, \sigma\}$. Then $u(x, t-\tau)>0$ and $u(x, t-\sigma)>0$ for $(x, t) \in \Omega \times\left(t_{0}, \infty\right)$. Multiply both sides of equation (1) by the eigenfunction $\varphi(x)$ of the Dirichlet problem and integrate with respect to $x$ over the domain $\Omega$. For $t \geqq t_{0}$ we obtain

$$
\begin{aligned}
& \frac{d^{2}}{d t^{2}}\left[\int_{\Omega} u(x, t) \varphi(x) d x+\lambda(t) \int_{\Omega} u(x, t-\tau) \varphi(x) d x\right]- \\
& \quad-\left[\int_{\Omega} \Delta u(x, t) \varphi(x) d x+\mu(t) \int_{\Omega} \Delta u(x, t-\sigma) \varphi(x) d x\right]+ \\
& \quad+\int_{\Omega} c(x, t, u) \varphi(x) d x=\int_{\Omega} f(x, t) \varphi(x) d x
\end{aligned}
$$


From Green's formula it follows that.

$$
\begin{aligned}
& \int_{\Omega} \Delta u(x, t) \varphi(x) d x=\int_{\Omega} u(x, t) \Delta \varphi(x) d x= \\
& =-\alpha_{0} \cdot \int_{\Omega} u(x, t) \varphi(x) d x=-\alpha_{0} w(t) \cdot \int_{\Omega} \varphi(x) d x \\
& \int_{\Omega} \Delta u(x, t-\sigma) \varphi(x) d x=\int_{\Omega} u(x, t-\sigma) \Delta \varphi(x) d x= \\
& =-\alpha_{0} \int_{\Omega} u(x, t-\sigma) \varphi(x) d x=-\alpha_{0} w(t-\sigma) \cdot \int_{\Omega} \varphi(x) d x
\end{aligned}
$$

where $\alpha_{0}$ is the smallest eigenvalue. Moreover, from condition H4 and Jensen's inequality it follows that

$$
\begin{array}{r}
\int_{\Omega} c(x, t, u) \varphi(x) d x \geqq p(t) \int_{\Omega} h(u) \varphi(x) d x \geqq \\
\geqq p(t) \cdot h\left(\int_{\Omega} u(x, t) \varphi(x) d x \cdot\left(\int_{\Omega} \varphi(x) d x\right)^{-1}\right) \cdot \int_{\Omega} \varphi(x) d x= \\
=p(t) \cdot h(w(t)) \cdot \int_{\Omega} \varphi(x) d x
\end{array}
$$

Using (16)-(18) and condition $\mathrm{H} 1$, from (15) we obtain

$$
\begin{aligned}
& \frac{d^{2}}{d t^{2}}[w(t)+\lambda(t) w(t-\tau)] \leqq-\alpha_{0}[w(t)+\mu(t) w(t-\sigma)]- \\
& -p(t) \cdot h(w(t))+\int_{\Omega} f(x, t) \varphi(x) d x \cdot\left(\int_{\Omega} \varphi(x) d x\right)^{-1}
\end{aligned}
$$

which completes the proof of Lemma 2.

Introduce the notation

$$
F_{1}(t)=\int_{\Omega} f(x, t) \varphi(x) d x \cdot\left(\int_{\Omega} \varphi(x) d x\right)^{-1}, \quad t>0
$$

Analogously to Theorem 1 the following theorem is proved.

Theorem 2. Let conditions $H 1-H 5$ hold and let the differential inequalities of neutral type

(20) $\frac{d^{2}}{d t^{2}}[w(t)+\lambda(t) w(t-\tau)]+\alpha_{0}[w(t)+\mu(t) w(t-\sigma)]+$

$$
+p(t) \cdot h(w(t)) \leqq F_{1}(t), \quad t \geqq t_{0}
$$




$$
\begin{aligned}
\frac{d^{2}}{d t^{2}}[w(t)+\lambda(t) w(t-\tau)]+ & \alpha_{0}[w(t)+\mu(t) w(t-\sigma)]+ \\
& +p(t) \cdot h(w(t)) \leqq-F_{1}(t), \quad t \geqq t_{0}
\end{aligned}
$$

have no eventually positive solutions. Then each solution $u(x, t)$ of problem (1), (3) oscillates in the domain $G$.

From the theorems proved above it follows that the finding of sufficient conditions for oscillation of the solutions of equation (1) in the domain $G$ is reduced to the investigation of the oscillatory properties of differential inequalities of neutral type of the form

$$
\begin{aligned}
& \frac{d^{2}}{d t^{2}}[x(t)+\lambda(t) x(t-\tau)]+q_{0}(t) x(t)+q(t) x(t-\sigma)+ \\
& +p(t) \cdot h(x(t)) \leqq H(t), \quad t \geqq t_{0}
\end{aligned}
$$

We shall say that condition (A) are satisficd if the following conditions hold:

A1. $\lambda(t) \in C^{2}\left(\left[t_{0}, \infty\right) ;[0, \infty)\right)$,

A2. $q_{0}(t), q(t) \in C\left(\left(t_{0,}, \infty\right) ;[0, \infty)\right)$,

A3. $p(t) \in C\left(\left(t_{0}, \infty\right) ;[0, \infty)\right)$,

A4. $h(u) \in C(\mathbb{R} ; \mathbb{R}), h(u)>0$ for $u>0$,

A5. $\left.H(t) \in C\left(\mid t_{0} ; \infty\right) ; \mathbb{R}\right)$.

Theorem 3. Let conditions (A) hold as well as the condition

$$
\liminf _{t \rightarrow \infty} \frac{1}{t-t_{1}} \int_{t_{1}}^{t}(t-s) \cdot H(s) d s=-\infty
$$

for $t_{1} \geqq t_{0}$. Then the differential inequality (22) has no eventually positive solutions.

Proof: Suppose that this is not true and let $x(t)$ be a positive solution of inequality (22) defined in the interval $\left(t_{1}, \infty\right)$, where $t_{1} \geqq t_{0}$. Then in virtue of conditions A2-A4 we obtain for $t \geqq t_{2}\left(t_{2} \geqq t_{1}+\max \{\sigma, \tau\}\right)$

$$
\begin{aligned}
\frac{d^{2}}{d t^{2}}[x(t)+\lambda(t) x(t-\tau)] \leqq H(t)-q_{0}(t) x(t) & -q(t) x(t-\sigma)- \\
& -p(t) \cdot h(x(t)) \leqq H(t) .
\end{aligned}
$$

We integrate twice the above inequality over the interval $\left[t_{2}, t\right]_{t} t>t_{2}$ and obtain

$$
x(t)+\lambda(t) x(t-\tau) \leqq C_{1}+C_{2}\left(t-t_{2}\right)+\int_{t_{2}}^{t}\left[\int_{t_{2}}^{\rho} H(s) d s\right] d \rho_{,}
$$


where $C_{1}, C_{2}=$ const. Since

$$
\int_{t_{2}}^{t}\left[\int_{t_{2}}^{p} H(s) d s\right] d \rho=\int_{t_{2}}^{t}(t-s) H(s) d s
$$

dividing both sides of last inequality by $t-t_{2}>0$, we obtain

$$
\frac{x(t)+\lambda(t) x(t-\tau)}{t-t_{2}} \leqq \frac{C_{1}}{t-t_{2}}+C_{2}+\frac{1}{t-t_{2}} \int_{t_{2}}^{t}(t-s) H(s) d s
$$

Then for $t \rightarrow \infty$ from (24), making use of condition (23), we obtain that

$$
\liminf _{t \rightarrow \infty} \frac{x(t)+\lambda(t) x(t-\tau)}{t-t_{2}}=-\infty
$$

On the other hand, using condition $\mathrm{A} 1$ and the fact that $x(t)>0, x(t-$ $\tau)>0$ for $t \geqq t_{2}$, we obtain that

$$
\liminf _{t \longrightarrow \infty} \frac{1}{t-t_{0}}[x(t)+\lambda(t) x(t-\tau)] \geqq 0
$$

which contradicts equality (25).

This completes the proof of Theorem 3 .

The following sufficient condition for oscillation of the solutions of problem (1), (2) is a corollary of Theorem 1 and Theorem 3.

Theorem 4. Let conditions (H) hold as well as the conditions

$$
\liminf _{t \rightarrow \infty} \int_{t_{0}}^{t}\left(1-\frac{s}{t}\right)(G(s)+\mu(s) G(s-\sigma)+F(s)) d s=-\infty,
$$

$$
\limsup _{t \rightarrow \infty} \int_{t_{0}}^{t}\left(1-\frac{s}{t}\right)(G(s)+\mu(s) G(s-\sigma)+F(s)) d s=+\infty
$$

for any sufficiently large number $t_{0}$, where the functions $G(t)$ and $F(t)$ are defined by (4). Then each solution $u(x, t)$ of problem (1), (2) oscillates in the domain $G$.

The following sufficient condition for oscillation of the solutions of problem (1), (3) is a corollary of Theorem 2 and Theorem 3 . 
Theorem 5. Let conditions H1-H5 hold as well as the conditions

$$
\mu(t) \geqq 0 \text { for } t \geqq 0
$$

$$
\begin{aligned}
& \liminf _{t \rightarrow \infty} \int_{t_{0}}^{t}\left(1-\frac{s}{t}\right) F_{1}(s) d s=-\infty \\
& \limsup _{t \rightarrow \infty} \int_{t_{0}}^{t}\left(1-\frac{s}{t}\right) F_{1}(s) d s=+\infty
\end{aligned}
$$

for any sufficiently large number $t_{0}$, where the function $F_{1}(t)$ is defined by (19). Then each solution $u(x, t)$ of problem (1), (3) oscillates in the domain $G$.

Example 1. Consider the equation

$$
\begin{array}{r}
u_{t t}+u_{t t}(x, t-\pi)-u_{x x}+u=2 e^{t} \cos x\left(\sin t+\cos t-e^{-\pi} \cdot \cos t\right) \\
(x, t) \in\left(0, \frac{\pi}{2}\right) \times(0, \infty) \equiv G
\end{array}
$$

and the boundary conditions

$$
-u_{x}(0, t)=0, \quad u_{x}\left(\frac{\pi}{2}, t\right)=-e^{t} \cdot \sin t, \quad t \geqq 0
$$

A straightforward verification shows that the functions

$$
\begin{aligned}
c(x, t, u) & =u, f(x, t)=2 e^{t} \cdot \cos x \cdot\left(\sin t+\cos t-e^{-\pi} \cos t\right), \\
g(0, t) & =0, g\left(\frac{\pi}{2}, t\right)=-e^{t} \cdot \sin t, \lambda(t) \equiv 1, \mu(t) \equiv 0, \\
\gamma(x, t) & \equiv 0
\end{aligned}
$$

satisfy conditions (H). Moreover, from (4) we obtain that

$$
\begin{aligned}
& G(t)=-\frac{2}{\pi} e^{t} \cdot \sin t, \quad t>0, \\
& F(t)=\frac{4}{\pi} e^{t}\left(\sin t+\cos t-e^{-\pi} \cdot \cos t\right), \quad t>0 .
\end{aligned}
$$

By straightforward calculations we find that

$$
\begin{aligned}
I(t)=\int_{t_{0}}^{t}\left(1-\frac{s}{t}\right)(G(s)+\mu(s) G(s-\sigma)+F(s)) d s= \\
=e^{t} \cdot(t \pi)^{-1} \cdot\left(2 \sin t-2 e^{-\pi \pi} \sin t-\cos t\right)+C,
\end{aligned}
$$


where $C$ is independent of $t$. Hence

$$
\liminf _{t \rightarrow \infty} I(t)=-\infty, \quad \limsup _{t \rightarrow \infty} I(t)=+\infty,
$$

i.e. conditions (26), (27) of Theorem 4 hold as well. Then from Theorem 4 it follows that each solution of problem (31), (32) oscillates in the domain $G=\left(0, \frac{\pi}{2}\right) \times(0, \infty)$. For instance, the function $u(x, t)=e^{t} \sin t \cos x$ is such a solution.

Example 2. Consider the equation

$$
\begin{aligned}
u_{t t}+u_{t t}(x, t-\pi)-\left[u_{x x}+u_{x x}(x, t-\pi)\right]+u & =f(x, t), \\
(x, t) & \in(0, \pi) \times(0, \infty) \equiv G,
\end{aligned}
$$

where $f^{\prime}(x, t)=e^{t} \cdot \sin x\left(2 e^{-\pi} \sin t-2 \sin t+e^{-\pi} \cos t\right)$ and the boundary conditions

$$
u(0, t)=u(\pi, t)=0, \quad t \geqq 0 .
$$

It is immediately verified that the functions

$$
c(x, t, u)=u, \quad f(x, t), \quad \lambda(t)=\mu(t)=1
$$

satisfy conditions H1-H5. Moreover, the smallest eigenvalue of the Sturrn-Lionville problem

$$
U^{\prime \prime}+\alpha U=0, \quad U(0)=U(\pi)=0
$$

is $\alpha_{0}=1$ and the corresponding eigenfunction is $\varphi(x)=\sqrt{\frac{2}{\pi}} \sin x>$ $0, x \in(0, \pi)$. Then from (19) we find that

$$
\begin{aligned}
F_{1}(t)=\int_{0}^{\pi} f(x, t) \sqrt{\frac{2}{\pi}} \sin x d x & \cdot\left(\int_{0}^{\pi} \sqrt{\frac{2}{\pi}} \sin x d x\right)^{-1}= \\
& =\frac{\pi}{4} e^{t}\left(2 e^{-\pi} \sin t-2 \sin t+e^{-\pi} \cos t\right) .
\end{aligned}
$$

By straightforward calculations we obtain that

$$
I_{1}(t)=\int_{t_{0}}^{t}\left(1-\frac{s}{t}\right) F_{1}(s) d s=\frac{\pi}{4} \cdot \frac{e^{t}}{t}\left(\cos t-e^{-\pi} \cos t+\frac{1}{2} e^{-\pi} \sin t\right)+C,
$$

where $C$ is indepondent of $t$. Hence $\liminf _{t \rightarrow \infty} I_{1}(t)=-\infty$ and $\limsup _{t \rightarrow \infty} I_{1}(t)=$ $+\infty$, i.e. conditions (29), (30) of Thcorem 5 hold as well. Then from 
Theorem 5 it, follows that each solution of probkern (33), (34) oscillates in the domain $G=(0, \pi) \times(0, \infty)$. For instance, the function $u(x, t)=$ $e^{t} \sin x \cdot \cos t$ is such a solution.

In the subsequent theorems we shall restrict our attention to some particular cases of equation (I) for which new sufficient conditions for oscillation of the solutions are obtained.

Case A.

Assume that $\lambda(t) \equiv 0$. We shall use the following result of $T$. Kusano and $M$. Naito [5] concerning differential inequalities of the form

$$
\left(q(t)(p(t) \cdot x)^{\prime}\right)^{\prime}+h(t, x) \leqq r(t), \quad t \geqq t_{0}
$$

We shall say that conditions (B) are satisfied if the following conditions hold:

B1. $p(t), q(t) \in C\left(\left[t_{0}, \infty\right) ;(0, \infty)\right), \int_{t_{0}}^{\infty}(q(t))^{-1} d t=\infty$.

B2. $h(t, x) \in C\left(\left[t_{0}, \infty\right) \times(0, \infty) ;(0, \infty)\right), h(t, x)$ is a monotone increasing function of its second argument $x$.

B3. $r(t) \in C\left(\left[t_{0}, \infty\right) ; \mathbb{R}\right)$.

Theorem 6 [5]. Let conditions (B) hold and let the differential inequality

$$
\left(q(t)(p(t) x)^{\prime}\right)^{\prime}+h(t, x) \leqq 0
$$

have no eventually positive solutions. Moreover, let a function $\theta(t) \in C^{2}\left(\left[t_{0}, \infty\right) ; \mathbb{R}\right)$ exist with the following properties:

(37) $\theta(t)$ takes both positive and negative values

for arbitrarily large values of $t$.

$$
\begin{gathered}
\left(q(t)(p(t) \cdot \theta(t))^{\prime}\right)^{\prime}=r(t), \quad t \geqq t_{0} \\
\operatorname{liminif}_{t \longrightarrow \infty}[p(t) \cdot \theta(t)]=0
\end{gathered}
$$

Then the differential inequality (35) has no eventually positime solutions.

The following sufficient condition for oscillation of the solutions of problem (1), (2) in the case when $\lambda(t) \equiv 0$ is a corollary of Theorem 1 and Theorem 6 . 
Theorem 7. Let the following conditions be fulfilled:

1. Conditions $(H)$ hold.

2. The function $h(u)$ is monotone increasing in the interval $(0, \infty)$.

3. The differential inequality

$$
x^{\prime \prime}(t)+p(t) \cdot h(x(t)) \leqq 0, \quad t \geqq t_{0}
$$

has no eventually positive solutions.

4. There exists a function $\theta(t) \in C^{2}\left(\left[t_{0}, \infty\right) ; \mathbb{R}\right)$ with the following properties:

a) $\theta(t)$ takes both positive and negative values for arbitrarily large values of $t$.

b) $[\theta(t)]^{\prime \prime}=G(t)+\mu(t) G(t-\sigma)+F(t), \quad t \geqq t_{1}$

c) $\lim _{t \rightarrow \infty} \theta(t)=0$.

Then each solution $u(x, t)$ of problem (1), (2) oscillates in the domain $G$.

Example 3. Consider the equation

(40) $u_{t \ell}(x, t)-u_{x x}(x, t)-u_{x x}(x, t-\pi)+2 u=f(x, t)$,

$$
(x, t) \in\left(0, \frac{\pi}{2}\right) \times(0, \infty)
$$

where $f(x, t)=e^{-t} \cdot \cos x \cdot\left(3 \sin t-2 \cos t-e^{\pi} \sin t\right)$ and the boundary condition

$$
-u_{x}(0, t)=0, u_{x}\left(\frac{\pi}{2}, t\right)=-e^{-\imath} \sin t, \quad t>0
$$

It is immediately verified that the functions

$$
\begin{array}{lrrr}
c(x, t, u)=2 u, & f(x, t), & \lambda(t) \equiv 0, \quad \mu(t) \equiv 1, \\
\gamma(x, t) \equiv 0, & g(0, t)=0, & g\left(\frac{\pi}{2}, t\right)=-e^{-t} \cdot \sin t
\end{array}
$$

satisfy conditions (H). Moreover, from (4) we obtain

$$
\begin{aligned}
& G(t)=-\frac{2}{\pi} e^{-t} \sin t, \quad t>0 \\
& F(t)=\frac{2}{\pi} e^{-i} \cdot\left(3 \sin t-2 \cos t-e^{\pi} \sin t\right), \quad t>0 .
\end{aligned}
$$

Then

$$
\begin{aligned}
I_{2}(t)=\int_{t_{0}}^{t}\left(1-\frac{s}{t}\right)(G(s)+\mu(s) G(s-\sigma)+F(s)) d s= \\
\quad=\int_{t_{0}}^{t}\left(1-\frac{s}{t}\right) \cdot \frac{2}{\pi} e^{-s} \cdot\left(3 \sin s-2 \cos s-e^{\pi} \sin s\right) d s
\end{aligned}
$$


which immediately implies that $\lim _{t \rightarrow \infty} I_{2}(t)<\infty$. Hence conditions (26) and (27) of Theorem 4 are not satisfied. It is easy to check that the differential inequality $x^{\prime \prime}+2 x \leqq 0$ has no eventually positive solutions. Let $\theta(t)=\frac{e^{-t}}{\pi}\left[2 \sin t+\left(3-e^{\pi}\right) \cos t\right]$. Then for $n \in \mathbb{Z}$ wo obtain

$$
\theta\left(\frac{\pi}{2}+2 n \pi\right)>0, \quad \theta\left(\frac{3 \pi}{2}+2 n \pi\right)<0 .
$$

Moreover, $[\theta(t)]^{\prime \prime}=\frac{2}{\pi} e^{-t} \cdot\left(3 \sin t-2 \cos t-e^{\pi} \sin t\right)=G(t)+G(t-$ $\pi)+F(t)$ and $\lim _{t \rightarrow \infty} \theta(t)=0$. Hence the function $\theta(t)$ satisfies condition 4 of Theorem 7 . Then by Theorem 7 each solution $u(x, t)$ of problem (40), (41) oscillates in the domain $G=\left(0, \frac{\pi}{2}\right) \times(0, \infty)$. For instance, the function $u(x, t)=e^{-t} \sin t \cos x$ is such a solution.

We shall note that a result analogous to that of Theorem 7 can be obtained for problern (1), (3) as well.

Case B.

Assume that $f(x, t) \equiv 0, g(x, t) \equiv 0$. In this case the finding of sufficient conditions for oscillation of the solutions of equation (1) in the domain $G$ is reduced to the investigation of the oscillatory properties of differential inequalitics of neutral type of the form

$$
\begin{aligned}
& \frac{d^{2}}{d t^{2}}[x(t)+\lambda(t) x(t-\tau)]+q_{0}(t) x(t)+q(t) x(t-\sigma)+ \\
& +p(t) \cdot h(x(t)) \leqq 0, \quad t \geqq t_{0}, \\
& \frac{d^{2}}{d t^{2}}[x(t)+\lambda(t) x(t-\tau)]+q_{0}(t) x(t)+q(t) x(t-\sigma)+ \\
& +p(t) \cdot h(x(t)) \geqq 0, \quad t \geqq t_{0} .
\end{aligned}
$$

Together with (42) and (43) we shall consider the nonlinear differential equation of neutral type

$$
\begin{aligned}
& \frac{d^{2}}{d t^{2}}[x(t)+\lambda(t) x(t-\tau)]+q_{0}(t) x(t)+q(t) x(t-\sigma)+ \\
& +p(t) \cdot h(x(t))=0, \quad t \geqq t_{0} .
\end{aligned}
$$

We shall say that conditions (C) are satisfied if the following conditions hold:

C1. $\lambda(t) \in C^{2}\left(\left[t_{0}, \infty\right) ; \mathbf{R}\right)$,

$$
0<\lambda_{1} \leqq \lambda(t) \leqq \lambda_{2} \text { for } t \geqq t_{0}, \lambda_{1}, \lambda_{2}=\text { const. }
$$

C2. $q_{0}(t), q(t) \in C\left(\left[t_{0}, \infty\right) ;[0, \infty)\right)$,

C3. $\left.p(t) \in C\left(\mid t_{0}, \infty\right) ;(0 ; \infty)\right)$,

C4. $h(u) \in C(\mathbb{R} ; \mathbb{R}), h(-u)=-h(u)$, 
$h(u)$ is a positive and monotone increasing function in the interval $(0, \infty)$.

Theorem 8. Let the following conditions be satisfied:

1. Conditions $(C)$ hold.

2. For any closed and measurable set $E \subset\left[t_{0}, \infty\right)$ for which meas $(E \cap[t, t+2 \tau]) \geqq \tau, t \in\left[t_{0}, \infty\right)$, the follouing condition holds

$$
\int_{E} p(t) d t=\infty
$$

Then:

(i) the differential inequality (42) has no eventually positive solutions;

(ii) the differentiol inequality (43) has no eventually negative solutions;

(iii) oll solutions of the differential equation (44) oscillate.

\section{Proof:}

(i) Let $x(t)$ be an eventually positive solution of the differential inequality (42). Then there exists a number $t_{1} \geqq t_{0}$ such that $x(t)>$ $0, x(t-\tau)>0$ and $x(t-\sigma)>0$ for $t \geqq t_{1}$. From conditions $\mathrm{C} 2-\mathrm{C} 4$ and (42) it folkows that

$$
\begin{aligned}
& \frac{d^{2}}{d t^{2}}[x(t)+\lambda(t) x(t-\tau)] \leqq-q_{0}(t) x(t)-q(t) x(t-\sigma)- \\
& -p(t) \cdot h(x(t)) \leqq-p(t) \cdot h(x(t))<0, \quad t \geqq t_{1}
\end{aligned}
$$

Hence the function $\frac{d}{d t}[x(t)+\lambda(t) x(t-\tau)]$ is monotone decreasing in the intcrval $\left[t_{1}, \infty\right)$. Suppose that there cxists a number $t_{2} \geqq t_{1}$ such that $\frac{d}{d t}\left[x\left(t_{2}\right)+\lambda\left(t_{2}\right) x\left(t_{2}-\tau\right)\right]=-c<0$. Then for arly point $t \geqq t_{2}$ the following inequality holds

$$
\frac{d}{d t}[x(t)+\lambda(t) x(t-\tau)] \leqq \frac{d}{d t}\left[x\left(t_{2}\right)+\lambda\left(t_{2}\right) x\left(t_{2}-\tau\right)\right]=-c
$$

Integrate last inequality over the interval $\left[t_{2}, t\right], t>t_{2}$ and obtain

$$
x(t)+\lambda(t) x(t-\tau) \leqq x\left(t_{2}\right)+\lambda\left(t_{2}\right) x\left(t_{2}-\tau\right)-c\left(t-t_{2}\right) .
$$

Hence lim sup $\{x(t)+\lambda(t) x(t-\tau)] \leqq 0$ which contradicts the assumption that $x(t)$ is an eventually positive solution. Hence

$$
\frac{d}{d t}[x(t)+\lambda(t) \cdot x(t-\tau)] \geqq 0, \quad t \geqq t_{1}
$$


whenee we obtain that $x(t)+\lambda(t) x(t-\tau) \geqq c_{1}>0$ for $t \geqq t_{1}$. From Lemma 1 [9], [11] it follows that there exists a closed and moisurable set $E \subset\left[t_{1}, \infty\right)$ and a constant $c_{2}>0$ such that $x(t) \geqq c_{2}$ for $t \in E$ and meas $(E \cap[t, t+2 \tau]) \geq \tau$ for $t \geqq t_{1}$. Then from condition C4 it follows that

$$
h(x(t)) \geqq h\left(c_{2}\right)=c_{3}>0 \text { for } t \in E .
$$

Integrate both sides of incquality (46) over the interva! $\left[t_{1}, t\right], t>t_{1}$ and using (47), wc obtain

$$
\begin{gathered}
C_{3} \int_{E \cap\left[t_{1}, t\right]} p(s) d s \leqq \int_{t_{1}}^{t} p(s) h(x(s)) d s \leqq \frac{d}{d t}\left[x\left(t_{1}\right)+\lambda\left(t_{1}\right) x\left(t_{1}-\tau\right)\right]- \\
-\frac{d}{d t}[x(t)+\lambda(t) x(t-\tau)] \leqq \frac{d}{d t}\left[x\left(t_{1}\right)+\lambda\left(t_{1}\right) x\left(t_{1}-\tau\right)\right]=C_{1} .
\end{gathered}
$$

For $t \longrightarrow \infty$ from the above inequality it follows that $\int_{E} p(t) d t<\infty$, which contradicts condition (45). Thus assertion (i) of Theorem 8 is proved.

(ii) The proof follows immediately from the fact that if $x(t)$ is an cventually negative solution of the differcrtial inequality (43), ther $-x(t)$ is an eventually positive solution of the differential inequality (42).

(iii) The proof follows imrnediately from assertions (i) and (ii).

The following sufficient condition for oscillation of the solutions of problem (1), (2) or (1), (3) in the case when $f(x, t) \equiv 0$ and $g(x, t) \equiv 0$ is a corollary of Theorem 1 , Theorem 2 and Theorem 8 .

Theorem 9. Let the following conditions hold:

1. Conditions $(H)$ are fulfilled.

2. $0<\lambda_{1} \leqq \lambda(t)<\lambda_{2}, t \geqq t_{0}, \lambda_{1}, \lambda_{2}=$ const.

3. $h(-u)=-h(u), u \in \mathbb{R} ; h(u)$ is a monotone increasing function in the interval $(0, \infty)$.

4. For any closed and measurable set $E \subset\left[t_{0}, \infty\right)$ for which meas $(E \cap\{t, t+2 \tau]) \geqq \tau, t \in\left[t_{0}, \infty\right)$ the following condition holds

$$
\int_{E} p(t) d t=\infty
$$

Then each solution $u(x, t)$ of problem (1), (2) or (1), (3) oscitlates in the domain $G$. 


\section{References}

1. V.S. Vladimirov, "Equations of Mathematical Physics," Moscow, Nauka, 1981 (in Russian).

2. D. Georgiou, K. KReITH, Functional characteristic initial value problems, J. Math. Anal. Appl. 107 (1985), 414-424.

3. D. GEORGIOU, "Extremal solutions of functional hyperbolic initial value problems, Differential equations: qualitative theory," vol. I, II (Szeged, 1984), Colloq. Math. Soc. János Bolyai 47, North-Holland, 1987.

4. K. KRirth, T. KusANo, N. Yosind, Oscillation properties of norlinear hyperbolic equations, Siam J. Math. Anal. 15, 3 (1984), $570-578$.

5. T. Kusano, M. NarTo, Oscillation criteria for a class of perturbed Schrödinger equations, Canad. Math. Bull. 25, 1 (1982), 71-77.

6. D.P. Misutev, Oscillatory properties of the solutions of hyperbolic differential equations with "maximum", Hiroshima Math. J. 16 (1986), 77-83.

7. D.P. Mishev, D.D. BAINov, Oscillation propertics of the solutions of a class of hyperbolic equations of neutra! type, Funkcialaj Ekvacioj 29 (1986), 213-218.

8. D.P. MisheV, D.D. BAINov, "Oscillation properties of the solutions of hyperbolic equations of neutral type, Differential equations: qualitative theory," vol. I, II (Szeged, 1984), 771-780, Colloc. Math. Soc. János Bolyai 47, North-Holland, 1987.

9. D.P. MisIIEV, Oscillation of the solutions of non-lincar parabolic cquntions of neutral type (to appear).

10. $\therefore$. Yosuliba, Oscillation of nonlinear parabolic equations with functional arguments, Hiroshima Math. J. 16, 2 (1986), 305-314.

11. A.I. ZahARIEV, D.D. BNNOV, Oscillating properties of the solutions of a class of neutral typc functional differential equations, Bull. Austral. Math. Soc. 22, 3 (1980), 365-372.

P.O. Box 45

1504 Sofia

BULGAIRIA

Rebut el 23 d'Agost de 1990 